\title{
Infection Control Measures for COVID-19 in the Labour Suite and Neonatal Unit
}

\author{
Pak Cheung $\mathrm{Ng}$ \\ Department of Paediatrics, Prince of Wales Hospital, Chinese University of Hong Kong, Hong Kong, China
}

In 2003, Hong Kong was ferociously attacked by a then unknown "pathogen" which overwhelmed the entire medical system within days and caused high casualties in healthcare workers (HCWs) and the general population $[1,2]$. Shortly after the outbreak, all medical disciplines gathered together to draw up an emergency infection control plan with a single objective - protection of patients and staff by preventing cross-infection. These emergency measures were merely based on prevailing medical knowledge and consensus of expert opinions rather than scientifically proven facts. Adopting and constantly updating these new guidelines, the Paediatric/Neonatal Unit at Prince of Wales Hospital, Hong Kong, experienced zero cross-infection between patients, and between patients and HCWs, and zero mortality with child patients and newborns [3,4]. Seventeen years later, a novel coronavirus SARS-CoV-2, returned with a vengeance [5] and the virus has spread across the world within a few months. Unlike SARS, infected subjects of COVID-19 could transmit the virus while being asymptomatic or having minimal symptoms, thus rendering the disease far more infectious and dangerous.

Despite the critical situation in Italy, Trevisanuto et al. [6] have done a magnificent job by summarizing key infection control measures against COVID-19 in this issue of Neonatology. However, as different maternal/neonatal units have different clinical and geographical setups and admission criteria, obstetricians and neonatologists should refer to these proposed measures as useful "recommendations," and individual units should feel free to fine-tune these recommendations according to their requirements or in light of new findings.

$\begin{aligned} & \text { karger@karger.com } \\ & \text { www.karger.com/neo }\end{aligned}$
Karger $\%$

\section{The Principles}

Two important fundamental principles have been repeatedly emphasized concerning maternal coronavirus infection during pregnancy. First, limited available evidence suggests that the coronavirus (SARS-CoV and SARS-CoV-2) is unlikely to be transmitted vertically from mothers to babies (even in preterm infants) [7], though recent reports indicated that newborns could acquire SARS-CoV-2 infection postnatally within days after birth. Secondly, maternal SARS or COVID-19 infection per se should not be an indication for early delivery. The timing and mode of delivery should solely be determined by the maternal respiratory status (e.g., progressive deterioration with increasing need for oxygen supplementation, escalation of ventilatory support due to increasing fetal size with splinting of diaphragmatic movement, and restriction of chest expansion in compromised infected lungs, resulting in respiratory failure), and obstetric indications.

\section{Effective Triaging}

Effective separation of COVID-19 patients from noninfected cases would be the key to success for prevention of cross-infection. It could be arranged at government level by determining which hospitals would be assigned to admit COVID-19 patients and which hospitals should only admit non-infected clean cases. This strategy would only be successful if large temporary hospitals were pur-

Prof. Pak C. NG, Clinical Professor (Honorary)

Department of Paediatrics, 6th Floor, Clinical Sciences Building

Prince of Wales Hospital

Shatin, N.T., Hong Kong (China)

pakcheungng@cuhk.edu.hk 


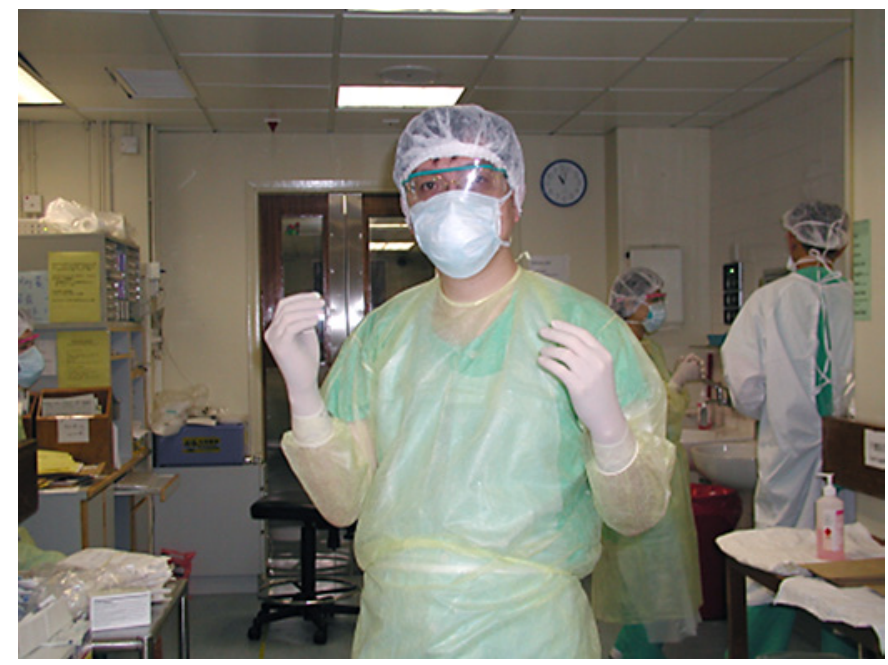

Fig. 1. A neonatologist (Prof. Pak C. Ng) in full personal protective equipment. With much sadness in his eyes, he found hope, comfort, and triumph over SARS within weeks after this photograph was taken in spring 2003.

posefully built for the pandemic. In most situations, hospitals would require admitting infected, suspected, and non-infected patients into different "clean" and "dirty" zones. The use of TOCC (Travel, Occupation, Contact and Cluster) history plus clinical features of respiratory and gastrointestinal symptoms could assist in the initial risk-stratified surveillance process. Unlike SARS in which the majority of infected patients presented with fever and definitive respiratory signs at the initial presentation, a substantial proportion of COVID-19 patients was actively shedding the virus asymptomatically and posed tremendous obstacles to the triaging process. The increasing use of the RT-PCR nucleic acid test for SARS-CoV-2 coupled with temporary segregation of high-risk cases (i.e., those with positive TOCC history or clinical features) into a "transitional ward" before the nucleic acid test results became available $(\sim 4-6 \mathrm{~h})$ might facilitate the triaging process. Nonetheless, the RT-PCR test has a sensitivity of about 0.70 , and additional investigations such as chest radiograph or high-resolution CT of thorax should also be considered for highly probable cases.

Since pregnant mothers with suspected or confirmed COVID-19 infection constitute only a very small number of overall admissions, an isolation room with negative pressure facilities can usually be arranged. If unavailable, one could consider opening windows to facilitate fresh air exchange in single rooms. In view of an increasing number of reports documenting early postnatal maternal- baby transmission, babies of mothers with suspected or confirmed COVID-19 would preferably be resuscitated in a separate room immediately after delivery and nursed in incubators in an isolation facility away from the mother until after 14 days and with repeated maternal RT-PCR tests confirmed negative. Daily video clips of infants' activities could be sent to parents to enhance mother-baby bonding. In addition, for any infant requiring positive pressure ventilatory support, nursing in an incubator would be recommended [4]. Since the setup of each maternal/neonatal unit is different, the final logistical arrangement would largely depend on its geographical and manpower constraints, but frontline neonatologists would still be recommended to adhere closely to principles of proper and effective triaging.

\section{Protection of Healthcare Workers}

Nothing could be more devastating and demoralizing than if members of the healthcare team are confirmed to be cross-infected. A few practical points are worth noting in the "warzone." Under normal environmental conditions, the coronavirus would be spread via droplets or through direct contact with fomites. In some special circumstances, the virus could travel a much longer distance simulating airborne transmission (e.g., use of nebulizer, vapour/aerosol-generating procedures during surgery or use of pressurized gas treatment). Proper protection with appropriate personal protective equipment would be mandatory during these procedures. The "gown-up" and "gown-down" areas must be separated to avoid contamination of equipment. The sequence of gowning-up (i.e., shoe covers $\rightarrow$ handwashing $\rightarrow$ N95 respirator $\rightarrow$ goggle or visor $\rightarrow$ cap $\rightarrow$ waterproof gown $\rightarrow$ handrub with $70 \%$ alcohol antiseptic agent $\rightarrow$ latex gloves; Fig. 1) and gowning-down (i.e., cap $\rightarrow$ gown $\rightarrow$ shoe covers $\rightarrow$ gloves $\rightarrow$ handwashing $\rightarrow$ goggle or visor $\rightarrow$ N95 respirator $\rightarrow$ handrub $\rightarrow$ new surgical mask) must be meticulously followed [4]. Big cartoon posters reminding HCWs of the sequences should be posted in the changing areas. From our previous experience, the "police nurse" designated to observe the proper gowning-up and gowning-down process of each HCW would be invaluable to ensure that the procedures were properly followed without accidental contamination [4].

Trevisanuto et al. [6] quite rightly point out that the number of HCWs allocated to each dirty team and the contact time between HCWs and suspected or confirmed COVID-19 patients should be kept to a minimum. A small team would enable more efficient deployment of 
HCWs and could space out the frequency of staff rotation into the dirty zone. In the worst scenario that a team was required to be quarantined, the number of HCWs affected could be kept to a minimum. It was also our practice to minimize the contact time between HCWs and highrisk patients by setting up vital sign monitors near the cubicle window/doorway (depending on the geographical constraints) or ideally having monitors connected to the central nursing station so that HCWs could observe the progress of infants without the need to enter the isolation area, except for performing physical procedures (e.g., endotracheal tube suction, orogastric tube feeding). Infants of COVID-19 mothers should have case note and observation chart ward rounds performed outside the isolation area, and the attending doctors could formulate a list of essential procedures that would require to be executed during patient contact (e.g., examining relevant organ systems, adjusting ventilator settings, altering infusion setting of drugs). However, we would not advocate routine periodic RT-PCR testing of asymptomatic HCWs with protected exposure at this stage, especially during a time of very limited resources worldwide.

\section{Summary}

Trevisanuto et al. [6] have pointed out many useful and practical points for the prevention of cross-infection, and thus, provide an important platform for individual obstetric and neonatal units to adapt their own infection control measures to accommodate their individual needs. In 2003, SARS gave Hong Kong and many Asian countries a painful but invaluable lesson $[1,2]$. Since then, simulation drills of life-threatening infectious diseases (e.g., SARS and Ebola) have been regularly conducted in most tertiary medical institutions. Stock checks of personal protective equipment were periodically updated. To date
(April 13, 2020), Hong Kong has 1,010 confirmed cases of COVID-19 and 4 deaths. Over $58 \%$ were imported cases associated with travellers and students who had already contracted the coronavirus overseas. The low incidence despite its close geographical proximity to mainland China could possibly be attributed to (1) the extremely high awareness of the population in the early phase of the outbreak (January 2020), (2) wearing of surgical face masks (>90\% population) in public areas [8], (3) frequent hand hygiene, (4) social distancing, and (5) strenuous contact tracing and quarantine measures by the health authorities in disrupting the infection chain. These actions prevented overwhelming and collapse of the medical system by the sheer reduction in the number of affected patients and allowed hospitals to operate in a manageable capacity. Unlike the Accident and Emergency and Internal Medicine departments, the number of pregnant COVID-19 mothers would not be high in comparison, and most frontline obstetricians and neonatologists were able to comply with most of these recommendations for protecting mothers, infants, and HCWs. My experience with SARS suggests that it would be the on-site experience, vigilant and meticulous clinical practice, plus frequent fine-tuning of existing infection control protocols in light of new information that would save you and your patients through this invisible but very deadly battle. With our concerted efforts, I have much hope that COVID-19 will be eradicated from our societies very soon.

\section{Disclosure Statement}

The author has no conflict of interest to declare.

\section{Funding Sources}

The author has no funding sources to declare.

\section{References}

1 Lee N, Hui D, Wu A, Chan P, Cameron P, Joynt GM, et al. A major outbreak of severe acute respiratory syndrome in Hong Kong. N Engl J Med. 2003 May;348(20):1986-94.

2 Hon KL, Leung CW, Cheng WT, Chan PK, Chu WC, Kwan YW, et al. Clinical presentations and outcome of severe acute respiratory syndrome in children. Lancet. 2003 May; 361(9370):1701-3.

3 Leung TF, Ng PC, Cheng FW, Lyon DJ, So $\mathrm{KW}$, Hon EK, et al. Infection control for SARS in a tertiary paediatric centre in Hong Kong. J Hosp Infect. 2004 Mar;56(3):215-22.
$4 \mathrm{Ng}$ PC, So KW, Leung TF, Cheng FW, Lyon DJ, Wong W, et al. Infection control for SARS in a tertiary neonatal centre. Arch Dis Child Fetal Neonatal Ed. 2003 Sep;88(5): F405-9.

5 Guan WJ, Ni ZY, Hu Y, Liang WH, Ou CQ, He JX, et al.; China Medical Treatment Expert Group for Covid-19. Clinical characteristics of coronavirus disease 2019 in China. N Engl J Med. 2020 Feb, Epub ahead of print.

6 Trevisanuto D, Moschino L, Doglioni N, Roehr CC, Gervasi MT, Baraldi E. Neonatal resuscitation where the mother has a suspect- ed or confirmed novel coronavirus (SARSCoV-2) infection: suggestion for a pragmatic action plan. Neonatology. 2020 Apr, Epub ahead of print.

7 Shek CC, Ng PC, Fung GP, Cheng FW, Chan $\mathrm{PK}$, Peiris MJ, et al. Infants born to mothers with severe acute respiratory syndrome. Pediatrics. 2003 Oct;112(4):e254.

8 Leung NH, Chu DK, Shiu EY, Chan KH, McDevitt JJ, Hau SJ, et al. Respiratory virus shedding in exhaled breath and efficacy of face masks. Nat Med. 2020. Available from: https://doi.org/10.1038/s41591-020-0843-2.
Infection Control Measures for COVID-19 in Maternal/Neonatal Units
Neonatology 2020;117:141-143 DOI: $10.1159 / 000508002$ 